\title{
Soft-Failure Localization and Time-Dependent Degradation Detection for Network Diagnosis
}

\author{
Sima Barzegar, Marc Ruiz, and Luis Velasco* \\ Optical Communications Group (GCO), Universitat Politècnica de Catalunya (UPC), Barcelona, Spain \\ *e-mail: barzegar@ac.upc.edu
}

\begin{abstract}
In optical networks, degradation of the Quality of Transmission (QoT) can be the outcome of soft-failures in optical devices, like Optical Transponders, Wavelength Selective Switches (WSS) and Optical Amplifiers (OA). In this paper, we assume time-dependent degradations on ROADMs and OAs. Specifically, several degradations are considered: $i$ ) the noise figure can increase linearly over time due to the aging of the components; $i i)$ the maximum of optical output power of the amplifiers can decrease because of the degradation in the pump lasers of the EDFAs; iii) aging effects, e.g., due to fiber splices; and $i v$ ) the OSNR can vary caused by frequency drift of WSSs due to temperature variations. Our proposal for degradation detection and soft-failure localization includes algorithms that are able to detect and localize the degradation in early stages and facilitate network diagnosis. In addition, we propose an architecture where the control plane consist of a network controller, a Monitoring and Data Analytics system and a QoT tool based on GNPy that are interconnected with each other.

Keywords: Soft-Failure Localization, Network diagnosis.
\end{abstract}

\section{INTRODUCTION}

In optical networks, degradation of the Quality of Transmission (QoT) can be the consequence of soft-failures in optical devices, like Optical Transponders (TRX), Wavelength Selective Switches (WSS) and Optical Amplifiers (OA). Those soft-failures can degenerate into hard-failures and affect a number of optical connections supporting a large amount of network services, and therefore it is of paramount importance not only to detect them as soon as possible [1], but also to localize the device causing the degradation to facilitate maintenance [2]. For such detection and localization to be possible, the control plane of the optical network needs to be enriched with Monitoring and Data Analytics (MDA) capabilities [3]. Once monitoring data have been collected from the data plane, data analytics algorithms can analyze them either as soon as they are available or periodically to proactively detect the degradation and anticipate hard-failures before they actually happen, and issue the proper recommendations to the network controller. The controller, in turn can make decisions about rerouting and/or reconfiguring the network, as well as to notify the management plane for scheduling maintenance.

Further, a considerable effort has been paying towards disaggregating the optical layer to enrich the offer of available solutions and to enable the deployment of optical nodes that better fit optical network operators' needs. However, such disaggregation tends to make network surveillance and maintenance more complex in general. In this context, some open-source projects, like GNPy, are considering the specific characteristics of the different optical devices that participate in the optical layer, like Reconfigurable Optical Add / Drop Multiplexers (ROADM), TRXs, and In-Line OAs, e.g., Erbium Doped Fiber Amplifier (EDFA). The GNPy library is being developed within the Telecom InfraProject for physical layer -aware networking [4]. The core of GNPy is the QoT estimator calculating the generalized signal to noise ratio (GSNR), considering both the ASE noise and Non-Linear Interference (NLI) accumulation, computed by means of the generalized Gaussian-noise model [5]. In order to derive the GSNR, a series of parameters is provided as input to the GNPy, together with the network topology, which includes the characteristics of the ROADMs, fiber types, span length, and EDFAs gain, power, and Noise Figure (NF). GNPy can be used as a tool to estimate the expected QoT for a set of lightpaths for several purposes, from off-line to in-operation network planning and re-optimization.

In this paper, we focus on scenarios of QoT degradation produced by soft-failures in optical devices and target not only at localizing the soft-failure, but also at estimating the evolution of the values of its working parameters that are causing the observed effects in the QoT. Note that this advanced network performance analysis procedure supported by GNPy tool facilitates diagnosis and network maintenance. Furthermore, because the relation between the monitored SNR and the value of the working parameters is nor linear, the analysis of the evolution of the later in the optical devices can accelerate degradation detection.

\section{SOFT-FAILURES ANALYSIS}

Several effects degrade the QoT within optical systems. Herewith, we focus on time-dependent degradations, a category which is also part of aging effects, which are usually taken into account by means of costly system margins. In this work, we considered degradation arising within the following modules: ROADMs and OAs that compensate entirely for the fiber losses within the span. A ROADM consists of WSSs and EDFAs, where the EDFAs are employed to recover for the filter insertion losses. 
Both building blocks face aging and non-ideal conditions. For example, although EDFAs are considered as robust devices, they also suffer aging or time-varying effects, as the increase of the NF over time due to the aging of the components. The NF is also frequency-dependent, and as the allocation of the spectrum might be time-dependent, the NF can result into a time-frequency variations. The pump lasers of the EDFAs also present degradation, which can be adjusted thanks to internal control loops, but which will still reduce the EDFA efficiency. For what concerns the WSSs, they might suffer temperature dependent variations, which might lead to frequency drift over time. Furthermore, as individual channels can drift as well, both effects can be highly detrimental in terms of QoT. In the context of this work, we consider the following time-varying device degradations: $a$ ) linear NF increasing over time; $b$ ) decrease of the maximum optical output power (P-max) of the amplifiers; $c$ ) the sudden variation of amplifier gain due optical restoration in the event of fiber cuts; and $d$ ) OSNR variations caused by frequency drifts of the WSSs due to temperature variations.

Our proposed architecture for soft-failure localization and device parameters estimation is shown in Fig. 1. The optical layer consists of a disaggregated set of ROADMs and TRXs, and a set of optical links interconnecting ROADMs with a number of OAs. The control plane includes $i$ ) a Network Controller to program the network devices; ii) a MDA system [3] that collates measurements from the data plane, analyses the data and issues recommendations to the network controller; and iii) a QoT tool based on GNPy estimates the SNR of the lightpaths and it is used for connection provisioning, as well as for diagnosis and failure localization.

The MDA controller collects measurements from the optical devices with a given periodicity; in this paper, we assumed the MDA collects SNR samples from the TRXs every 15 minutes. These measurements are compared to the GSNR values estimated by the QoT tool for every lightpath to check whether there is a meaningful deviation (threshold violation); in such case, a procedure called MESARTHIM-T is executed with the lightpaths exceeding the threshold. MESARTHIM-T analyzes the behavior of the measured SNR evolution to find correlation in the selected lightpaths. In addition, a sister algorithm called MESARTHIM-P is periodically run considering all the lightpaths in the network to identify anomalous behavior in the evolution of the estimated configuration parameters of the devices. Both focus on diagnosing soft-failures in the network.
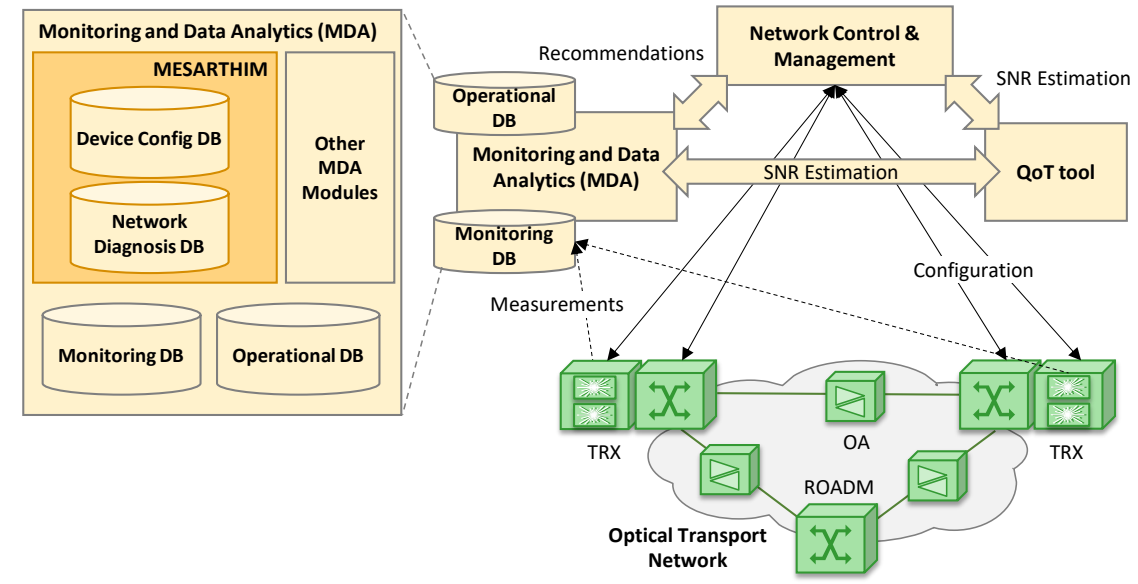

Fig. 1. Overview of the proposed surveillance architecture

The pseudocode of the MESARTHIM-T algorithm is presented in Table I, which receives the network graph $G$, the list of lightpaths $P$, the number $T$ of historical monitoring samples, and the connection to the QoT tool $Q$. The algorithm initializes the sets of found clusters $C$ that will capture the behavior observed in the lightpaths and $S C$ with the common resources supporting those lightpaths (line 1 in Table I). Next, the algorithm follows a twostep approach. First, it finds clusters of paths with a similar behavior (lines 2-5); for such analysis, the last $T$ monitoring samples are considered. Note that by considering the evolution of paths' SNR spurious measurements in one lightpath can be detected and ignored. (line 6). In the case that a set of lightpaths presents a similar anomalous behavior, MESARTHIM-T proceeds with the second step and finds the common resources on which those lightpaths are supported (line 8); for each common resource a likely evolution of the working parameters is found (lines 10-14). To find a likely device configuration, the QoT tool is interrogated with different values of the parameters and the configuration entailing the lowest SNR mean squared error for the set of lightpaths is returned. The resources, each with a subset of lightpaths and a configuration evolution, are eventually returned (line 15).

The pseudocode of the MESARTHIM-P algorithm is shown in Table II; it focuses on analyzing the behavior of the working parameters evolution of every resource in the network. Note that this analysis could detect softfailures that had not yet an impact on the lightpaths, as working parameters and SNR are not linearly related.

Table I. MESARTHIM-T Algorithm Pseudocode

\begin{tabular}{|l|l|}
\hline INPUT: $G, P, T, Q$ & OUTPUT: $S C$ \\
\hline
\end{tabular}




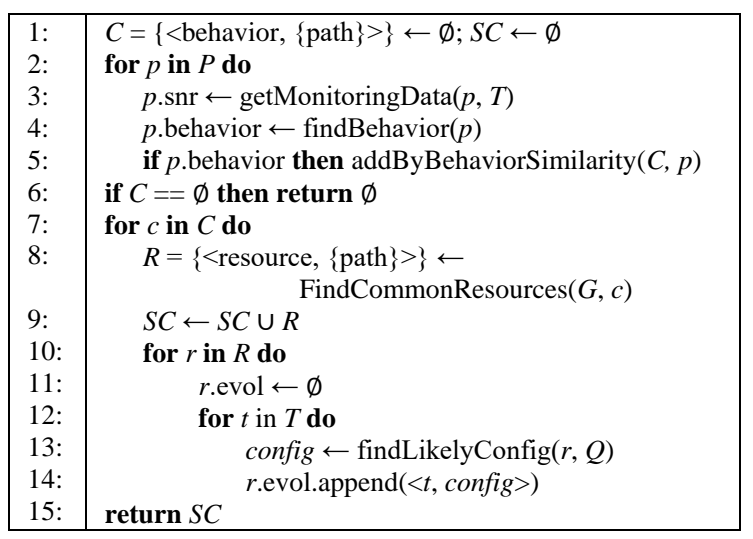

Table II. MESARTHIM-P Algorithm Pseudocode

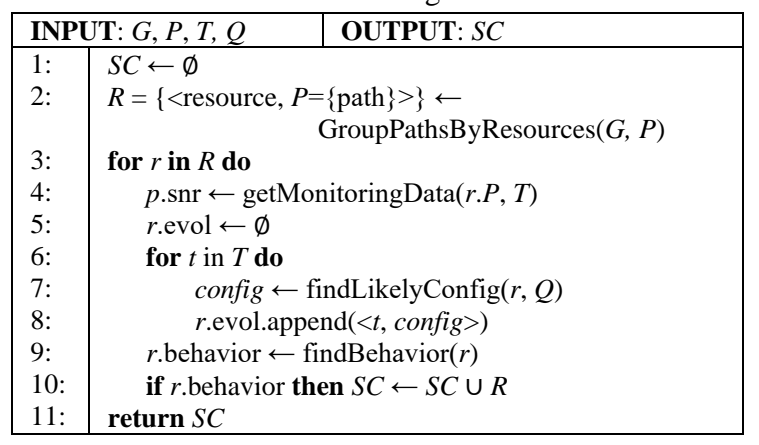

\section{RESULTS}

The architecture in Fig. 1 and the proposed procedures for soft-failure localization and device parameters estimation has been evaluated through simulation. A German-like network topology with 17 nodes and 52 links and 272 lightpaths representing all the origin-destination pairs was implemented, where a monitoring generator based on GNPy exported SNR measurements based on the value of working parameters. We selected different types of failures affecting an OA and an add/drop (A/D) WSS in a ROADM by forcing the working parameters of the selected devices (NF and P-max in the OAs and OSNR in the WSSs) to vary over time. From the different variations that might happen, we selected three to illustrate the performance of the proposed algorithms: gradual and periodical variations, and short-lived random events.

The graphs in the upper row in Fig. 2a-d present the evolution of the SNR over time monitored on a lightpath for the selected failures. Note that only the lightpaths affected by the failure will get their SNR evolving, whereas the rest of the lightpaths will show no variation over the time, other than a random one plus some uncorrelated spurious measurements introduced by the monitoring generator. Note that the time in the graphs in Fig. 2 has been normalized, as the time-scales for the considered soft-failures are different, ranging from days to years. SNR samples were stored in the simulated control plane and fed a module to decide whether the received value is anomalous as compared to the expected SNR for the corresponding lightpath, based on a programmable threshold; the threshold was set to a value that exceeds the random variations introduced by the monitoring generator. In the case of threshold violation, the MESARTHIM-T procedure was run with all the lightpaths exceeding the SNR threshold. In addition, the MESARTHIM-P procedure is run periodically with all the lightpaths in the network.

The evolution of the working parameters are shown in the graphs in the bottom row in Fig. 2a-d, where the actually programmed value of the parameter and the interval of values [max, min] estimated by the findLikelyConfig() function in the MESARTHIM procedures (lines 11-14 in Table I and lines 5-8 in Table II) are plotted. Several conclusions can be drawn from the results in Fig. 2. First, the accuracy of the proposed method for estimating the working parameters of the devices is very high for all three variations selected. In general, the estimation interval is tighter when the impact of the value of the parameter on the observed SNR is higher. In the specific case of the maximum power of the EDFAs, the range of values that result in the SNR values observed is large when the observed SNR remains constant around a normal value. However, as soon as a degradation is observed, the estimated interval becomes tight. 



Fig. 2. Evolution of monitored lighpath SNR with time and estimation of device working parameters.

Let us now compare the performance of the MESARTHIM procedures. The localization of soft-failures in an optical link or in a ROADM requires that several lightpaths are evaluated to find the common resource in the network topology. When the evolution of the monitored SNR changes suddenly, MESARTHIM-T collects enough lightpaths to easily localize the failure. Nonetheless, under a gradual degradation the lightpaths exceeding the threshold might be not enough for the localization. This is not the case for MESARTHIM-P, which by analyzing the estimated evolution of the working parameters by resource, it is able to detect degradations in their very early stages. The obtained detection times are highlighted with a red dot in Fig. 2a and $\mathrm{d}$, where the anticipation achieved by analyzing the evolution of the working parameters is remarkable.

\section{CONCLUSION}

The combined analysis of the evolution of the monitoring data and their transformation into the estimated working parameters, noticeably anticipates degradation detection and facilitates network diagnosis.

\section{ACKNOWLEDGEMENTS}

The research leading to these results has received funding from the Spanish MINECO TWINS project (TEC2017-90097-R), and from the Catalan Institution for Research and Advanced Studies (ICREA).

\section{REFERENCES}

[1] A. P. Vela et al., "BER Degradation Detection and Failure Identification in Elastic Optical Networks," IEEE/OSA Journal of Lightwave Technology (JLT), vol. 35, pp. 4595-4604, 2017.

[2] A. P. Vela et al., "Soft Failure Localization during Commissioning Testing and Lightpath Operation," IEEE/OSA Journal of Optical Communications and Networking (JOCN), vol. 10, pp. A27-A36, 2018.

[3] L. Velasco et al., "Monitoring and Data Analytics for Optical Networking: Benefits, Architectures, and Use Cases," IEEE Network Magazine, vol. 33, pp. 100-108, 2019.

[4] M. Filer et al., "Multi-vendor experimental validation of an open source QoT estimator for optical networks," IEEE/OSA Journal of Lightwave Technology (JLT), vol. 36, pp. 3073-3082, 2018.

[5] M. Cantono et al. "On the interplay of NL interference with stimulated Raman scattering for QoT estimation," IEEE/OSA Journal of Lightwave Technology (JLT), vol. 36, pp. 3131-3141, 2018. 